\title{
Pratiques
}

Linguistique, littérature, didactique

173-174| 2017

Le déjà-là dans l'écriture

\section{La production écrite vue comme un processus bilingue : dans quelle mesure les TIC peuvent-elles aider?}

Written production as a bilingual process: how far can ICT help?

Krastanka Bozhinova, Jean-Paul Narcy-Combes et Sonia Zaouali

\section{OpenEdition}

Journals

Édition électronique

URL : http://journals.openedition.org/pratiques/3426

DOI : $10.4000 /$ pratiques.3426

ISSN : 2425-2042

Éditeur

Centre de recherche sur les médiations (CREM)

Référence électronique

Krastanka Bozhinova, Jean-Paul Narcy-Combes et Sonia Zaouali, « La production écrite vue comme un processus bilingue : dans quelle mesure les TIC peuvent-elles aider? », Pratiques [En ligne],

173-174 | 2017, mis en ligne le 10 mars 2017, consulté le 19 avril 2019. URL : http://

journals.openedition.org/pratiques/3426 ; DOI : 10.4000/pratiques.3426

Ce document a été généré automatiquement le 19 avril 2019.

(c) Tous droits réservés 


\section{La production écrite vue comme un processus bilingue : dans quelle mesure les TIC peuvent-elles aider?}

Written production as a bilingual process: how far can ICT help?

Krastanka Bozhinova, Jean-Paul Narcy-Combes et Sonia Zaouali

1 Écrire en Langue 2 (L2) démoralise souvent les apprenants qui ont parfois le sentiment de ne pas pouvoir "penser » dans cette langue comme si rien n'était déjà là. Initialement, lire que les codes disponibles ne sont jamais totalement désactivés (Herdina \& Jessner, 2002) nous a conduits à faire appel à l'alternance codique pour déclencher la production, et il nous a semblé judicieux de recourir aux outils que proposent les technologies de l'information et de la communication (TIC). Cet article synthétisera deux expériences de recherche-action qui avaient, dans des contextes différents, des objectifs pratiques très proches. Il suivra un plan canonique (contextes, méthodologie, données et analyses), pour aboutir à une discussion des apports communs des expériences et de leurs limites.

\section{Point de départ}

2 Au sujet de l'écrit, nous nous demandions s'il n'était pas possible d'introduire des étapes entre la créativité (qui peut être conscientisée en langue source: le déjà-là) et la production en langue cible. Comme de nombreux enseignants, nous souhaitions pouvoir entrainer et guider l'apprenant dans un exercice qui reste individuel et qui n'est pas dans l'immédiateté. Nous voulions aussi lui permettre d'être plus autonome en utilisant des outils à sa portée et en développant des stratégies visant une utilisation raisonnée des traducteurs, dictionnaires et outils en ligne pour améliorer sa production écrite (PE). Nous étions également sensibilisés au travail collaboratif d'écriture (Lamy \& Hampel, 2007) et cherchions à en intégrer les aspects positifs dans nos pratiques.

3 Une expérience préalable sur l'emploi du copier-coller pour rédiger des condensés de texte nous confortait dans notre intention. Elle avait été motivée par des recherches qui 
montraient que surligner les parties importantes d'un texte facilitait la mémorisation et le réemploi (Eisele-Hendersen, 2000). Selon D. Gaonac'h (1990), une attention trop grande aux processus de bas niveau gêne le travail de haut niveau, générant un manque de créativité, des erreurs, une mauvaise mémorisation de l'input ${ }^{1}$. L'ordinateur et le traitement de texte permettant d'importer un article et d'en rédiger un condensé en copiant/collant les éléments les plus pertinents de l'article (ce qui correspond à surligner), nous faisions donc l'hypothèse que (1) les textes de départ serviraient d'input et (2) la rédaction de condensés, en générant de l'output compréhensible, assurerait l' intake (Swain \& Lapkin, 1995). L'expérience nous a permis de développer une méthodologie pour guider les étudiants (Narcy-Combes, 2005). Des pré et post tests (ibid.) ont révélé des acquisitions mesurables en syntaxe, lexique et des gains en créativité. Les théories sur le fonctionnement langagier qui postulent une double forme de la production langagière confirment la pertinence de ce mode de travail (Logan, 1988 ; Ellis, 1993).

Deux déclencheurs nous ont incités à aller plus loin :

- le besoin de favoriser la créativité afin de parvenir à d'autres types de textes ;

- la rencontre avec la critique génétique (cf. Biasi, 2011) qui souligne la multimodalité et la complexité des brouillons des auteurs.

Des pistes théoriques nouvelles qui vont être détaillées maintenant s'ouvraient également à nous. Elles interpellaient la conception monolingue de l'écriture qui dominait jusque-là.

\section{Cadre théorique}

Dans la mesure où nous nous adressons à des apprenants pour qui l'écrit est déjà là, en langue 1, mais aussi sur le plan méthodologique, ce sont les aspects liés à la multiplicité des codes qui nous ont arrêtés. À la lumière d'approches psycholinguistiques (Herdina \& Jessner, 2002; Randall, 2007, entre autres) et de la réflexion sur le translanguaging que nous avons rencontrée chez C. Williams (2002) et O. Garcia (2009), nous avons postulé que le scripteur peut débuter par une écriture en deux étapes apparemment successives mais de plus en plus imbriquées. Initialement, il devrait avoir recours au déjà-là, aux matériaux à sa disposition, en particulier aux codes disponibles dans son répertoire, ainsi qu'à d'autres supports et à la pratique de l'écriture qu'il a développée. Il restera à déterminer la façon dont il utilisera les outils à sa disposition ou fera usage de l'intertextualité à partir de corpus mis à sa disposition.

7 Nous nous sommes appuyés sur :

- S. Williams \& B. Hammarberg (1998) qui distinguent deux rôles joués par les langues/ cultures 2, lors de l'acquisition d'une troisième langue. Une langue active au cours du processus de communication en L3 peut jouer soit un rôle instrumental, soit un rôle fournisseur. Selon nous, le rôle instrumental permet de gérer la réflexion et le rôle fournisseur la créativité.

- P. Herdina \& U. Jessner (2002), pour qui la distinction entre alternance codique et transfert n'est pas validée au plan méthodologique. Le transfert, le mélange de codes et l'alternance codique sont différentes positions sur un même continuum, ce qui conduit à postuler que toute production langagière implique l'activation partielle d'une autre langue que celle attendue avec adaptation au fonctionnement de la langue cible plus ou moins opératoire. Il importe alors que l'individu vérifie les résultats d'une telle production. 
De ce fait, le translanguaging semblait « un moyen naturel de développer et de renforcer les deux langues tout en approfondissant la compréhension ${ }^{2} »$ (Williams, 2002, p. 29). Nous avons gardé en mémoire que la multicompétence langagière n'est pas dissociable des contenus véhiculés dans des contextes variables (Narcy-Combes, 2005), ce qui peut avoir joué un rôle dynamisant dans un des contextes. Il restait à savoir comment nous pourrions favoriser la PE des apprenants de français (L2 ou L3) et en particulier, le développement des dimensions discursivo-pragmatiques.

9 Nos recherches (Bertin, Gravé \& Narcy-Combes, 2010) conduisent à penser qu'un dispositif fondé sur une approche par des tâches qui font sens dans la réalité sociale est susceptible de favoriser le développement à condition :

- d'articuler les activités de compréhension et de production écrite, en assurant le traitement cognitif d'un input dont on augmente la quantité ;

- de concilier les deux modes de production : par règles et par blocs lexicalisés (Wray, 2008; Narcy-Combes, 2005 ; Ellis, 1993 ; Logan, 1988);

- de recourir aux outils TIC qui permettent la collaboration et l'utilisation de ressources dans les langues connues par les apprenants.

Une expérience initiale a été mise en place avec des élèves français (Bussière, 2013), suivie de reprises dans des établissements scolaires et universitaires en Bulgarie, au Maroc et en Tunisie. Seules les expériences bulgare et tunisienne seront développées ici.

\section{Objectif et problématique}

11 L'objectif est de mener un projet d'encadrement/accompagnement des pratiques d'écriture propres aux domaines des apprenants. Dans l'expérience tunisienne, la fonction de l'écrit en L2 ou L3 est de s'approprier puis de transmettre des savoirs dans une discipline, tout en maitrisant un niveau de langue qui permette de rédiger des écrits disciplinaires conformes à des normes qui relèvent d'enjeux scolaires et sociaux. D'où l'intérêt d'aller au-delà de la langue discipline (Mangiante \& Parpette, 2011) pour réfléchir en termes de langue, ici français, sur objectifs universitaires (FOU). Le contexte bulgare avait des objectifs plus généraux. Les pratiques proposées reflètent donc ces différences essentielles, ce qui peut avoir influencé les résultats.

Pour mesurer la réussite des expériences, il fallait déterminer des indicateurs de développement langagier. Dans la mesure où les recherches étaient menées principalement sous la forme de recherche-action, il était impossible de mettre en place un dispositif quasi-expérimental, ce qui, en outre, aurait été déontologiquement délicat. Nous avons choisi de nous assurer que les travaux des apprenants favorisaient un développement en vérifiant la présence :

1. de séquences potentiellement acquisitionnelles (SPA) (de Pietro, Matthey \& Py, 1989). Nous avons fait l'hypothèse que l'écriture collaborative et le feedback de l'enseignant fourniraient aux apprenants des occasions de produire des énoncés non conformes aux normes attendues qui seraient repris par la tutrice, les pairs ou les destinataires, et que ces énoncés se retrouveraient ultérieurement formulés selon la reprise,

2. d'output compréhensible où se manifestent des éléments issus des sources d'input proposées (Swain \& Lapkin, 1995), et

3. d'implication puisque, depuis V. Cook (2008), nous savons que cette dernière est corrélée avec le développement. 
Il restait à déterminer comment mesurer la créativité. Deux pistes s'offraient à nous :

- une analyse quantitative selon l'hypothèse que plus on a d'idées et plus on écrit, ce qui conduit à comparer la longueur (en mots) des différents travaux en la reliant avec le nombre de thèmes repris, car la réduction des paraphrases peut donner des textes plus courts mais plus riches et plus complexes ;

- plus on s'implique et plus on développe ses idées, plus on rajoute de détails. Par conséquent on a besoin d'organiser davantage le texte. Ce qui conduit à observer les connecteurs logiques, la présence de "péripéties » et de détails dans le récit/texte, mais également le respect des pratiques pragmatico-discursives d'une communauté.

Ce positionnement a été adapté au contexte de chaque expérience.

\section{Contextes} Supérieur d'Informatique (ISI) de Tunis dont la validation est diplômante. La référence au FOU, en tant qu' « approche centrée sur la connaissance la plus poussée des besoins d'un public ciblé » (Mangiante \& Parpette, 2011), n'est pas sans incidence, puisque les exigences formelle, discursive, rhétorique et linguistique sont aussi importantes que le savoir et que, de surcroit, les cours de FOU développent des compétences méthodologiques (Goes, Mangiante \& Bordo, 2016).

L'échantillon comporte six étudiants du cycle ingénieur en $3^{\mathrm{e}}$ année, toutes spécialités confondues, et trois étudiants de M2 Sécurité des Systèmes Informatiques Communicants et Embarqués. Le groupe pilote a utilisé l'espace de cours personnel, créé sur Moodle 2.7 ( http://ent.uvt.rnu.tn/course/view.php?id=333).

\section{Mise en place des expériences}

Au-delà des différences contextuelles, chaque expérience avait ses exigences propres et des objectifs aux ambitions fort différentes.

En Bulgarie, l'écriture est vue comme un processus récursif, réflexif et sociocollaboratif, lié à l'exposition à des corpus de textes-ressources en ligne relevant des genres travaillés. Ainsi, une partie des PE ont fait l'objet de rédaction en binôme/petit groupe, de révisions successives à l'aide du feedback de l'enseignante-tutrice, le plus souvent en mode asynchrone, et d'outils multilingues d'aide à l'écriture (les traducteurs automatiques Google, Reverso et Babylon, les dictionnaires contextuels Linguee et Reverso Context, les 
correcteurs BonPatron, Reverso et Word, des conjugueurs et des dictionnaires connus par les apprenants). Ces PE faisaient partie de tâches sociales et étaient destinées à des partenaires locuteurs natifs ou menaient à la socialisation en ligne. D'autres PE étaient des tâches d'entrainement en binôme sur certains éléments langagiers.

Les tâches suivantes étaient proposées :

- Un pré-test réalisé au laboratoire de langues: attentes concernant le cours de français envoyées par courriel au professeur ;

- deux tâches sociales de longue durée impliquant la participation de locuteurs natifs réalisées en mode hybride sur la plateforme Elearn et par courriel: recueil de témoignages d'étudiants en programme d'échange et de stagiaires concernant la vie sur le campus et rédaction d'un article pour blog; envoi de lettres de demande d'information aux Centres de Français Langue Étrangère (FLE) en France au sujet de leur offre de cours d'été ;

- trois tâches d'entrainement avec des outils d'aide à la rédaction et à la révision en ligne faites en présentiel en temps limité et enregistrées sur Google Drive ;

- deux tâches réalisées lors des examens sur table à la fin des semestres sans outils ;

- une tâche de PE accompagnée de réflexion à haute voix, réalisée par certains des apprenants et enregistrée en vidéo à l'aide du logiciel de capture d'écran Microsoft Expression Encoder Screen Capture 4.0.

En Tunisie, le dispositif hybride d'aide à l'écriture/réécriture implique la réalisation d'une macrotâche consistant en la réécriture de rapports de Projets de fin d'études (PFE) et de mémoires de Mastère. Les apprenants sont appelés à :

- produire en reformulant, paraphrasant, enchainant et révisant leurs écrits avec les logiciels de correction intégrés (Word) ou en ligne (Cordial, Antidote...);

- citer des sources et évaluer l'originalité des textes réécrits grâce au logiciel d'antiplagiat Viper ou autres ;

- respecter des normes bibliographiques de la discipline en recourant à des générateurs bibliographiques intégrés ou en ligne ;

- s'autoévaluer/coévaluer en menant une réflexion métacognitive individuelle et collective dans un forum de discussion;

- résumer en plusieurs langues (emploi d'outils de traduction automatique en ligne).

Des séances d'encadrement ont été programmées en présentiel pour permettre une discussion des difficultés rencontrées lors de l'élaboration des écrits et une re-médiation (seconde médiation) collective ou personnalisée. La partie distancielle s'appuie sur le tutorat comme aide à l'élaboration de versions successives des écrits par révision, soulignement, commentaires et rétroaction. La médiation a dicté des logiques d'intervention selon trois postures (Ciekanski, 2014):

- une posture normative où des normes sont prescrites ;

- une posture dialogique où le discours de la tutrice dévoile les processus cognitifs et les savoirs que les étudiants mobilisent pour écrire ;

- une posture spéculative où l'empathie de la tutrice permet de sensibiliser l'étudiant à l'observation des processus mis en œuvre.

En raison de la complexité des contextes, de la diversité des tâches et des outils, les expériences permettaient un recueil de données d'une grande richesse. 


\section{Données recueillies} versions successives des PE. Parallèlement, sont relevées les SPA, c'est-à-dire la présence de rétroactions correctives et de reprises grâce au repérage et au recul déclenchés par le système d'accompagnement et de ressources. Les traces de ces séquences sont étudiées dans (1) les dialogues entre l'apprenant et l'enseignant-tuteur surtout en mode asynchrone, (2) la comparaison de l'input et des PE et (3) les entretiens semi-directifs.

\subsection{Développement langagier}

Le tableau 1 résume quelques exemples de développement langagier.

Tableau 1. Résumé des points principaux de développement observé

\begin{tabular}{|l|l|}
\hline $\begin{array}{l}\text { Éléments langagiers } \\
\text { ayant évolué }\end{array}$ & Évolution observée \\
\hline $\begin{array}{l}\text { expression de la demande } \\
\text { polie }\end{array}$ & $\begin{array}{l}\text { mise en place de la demande indirecte; conditionnel présent utilisé } \\
\text { plus correctement pour exprimer une demande polie }\end{array}$ \\
\hline
\end{tabular}




\begin{tabular}{|l|l|}
\hline connecteurs & $\begin{array}{l}\text { augmentation de la fréquence et enrichissement du répertoire pour } \\
\text { assurer la cohérence et organiser les textes }\end{array}$ \\
\hline $\begin{array}{l}\text { formules d'adresse et } \\
\text { formules finales dans les } \\
\text { courriels }\end{array}$ & $\begin{array}{l}\text { enrichissement du répertoire utilisé et adéquation au contexte ; } \\
\text { réutilisation de l'input avec adaptation }\end{array}$ \\
\hline $\begin{array}{l}\text { formules et techniques pour } \\
\text { rapporter les paroles } \\
\text { d'autrui }\end{array}$ & $\begin{array}{l}\text { enrichissement du répertoire de formules utilisées (expressions } \\
\text { respect des normes de citation et de reformulation } \\
\text { respelles; constructions verbales variées); meilleur }\end{array}$ \\
\hline $\begin{array}{l}\text { lexique, orthographe, } \\
\text { morphosyntaxe }\end{array}$ & $\begin{array}{l}\text { enrichissement selon le sujet traité, plus de correction et meilleure } \\
\text { adéquation }\end{array}$ \\
\hline
\end{tabular}

31 L'analyse des courriels des tâches sociales a permis d'observer le développement de l'expression de la demande. Le taux des occurrences d'expressions indirectes complexes n'augmente pas (autour de $45 \%$ ) mais une évolution qualitative importante est observée : passage d'expressions proches des L1 et L2 vers l'utilisation d'expressions plus adéquates. Par exemple, les premières formulations, telles que « Nous vous écrivons pour demander d'information sur... » sont remplacées par des expressions des banques de textes, comme "Nous aimerions savoir combien de jours... ». De plus, le taux des expressions révélant l'activation d'autres langues diminue de $41 \%$ à $34 \%$. Par conséquent, le discours des apprenants commence à se rapprocher de celui qui est attendu.

La fréquence des occurrences de connecteurs dans les dernières PE a augmenté et ils sont réemployés de manière nuancée selon le contexte. Les premiers courriels ne contenaient que des questions juxtaposées, des connecteurs non-conformes, «tout d'abord» et « aussi ». Dans les dernières PE, les étudiants ont utilisé des constructions plus complexes, telles que "en premier lieu/en deuxième lieu», «en outre», «d'autre part", « d'ailleurs », « ainsi que », « également ». L'activation de l'anglais reste évidente dans des expressions comme «en première place/en seconde place» (pour « en premier lieu/en second lieu »); « suivant » (équivalent à next), l'orthographe anglicisée (" par example ») ou encore la place de « aussi » (analogique à also).

L'adéquation des formules d'adresse s'est améliorée également. Les problèmes récurrents surmontés sont liés au mélange des registres, à l'emploi de formules trop formelles ou au contraire, trop familières pour les contextes respectifs. Par contre, l'emploi des formules finales n'est pas stabilisé, mais on constate un rapprochement du registre attendu.

En ce qui concerne le discours rapporté, au début, les apprenants se servaient presque exclusivement du verbe «dire » pour introduire les paroles d'autrui. L'entrainement à l'aide de lignes de concordance a favorisé le repérage des expressions et des verbes introductifs (8 des 14 formes ont été réutilisées). L'orthographe et la morphologie verbale se sont améliorées également. Le feedback visait à sensibiliser les apprenants aux normes de citation et de reformulation et renvoyait parfois à des outils en ligne pour observer l'emploi en contexte, comme dans l'exemple suivant ${ }^{3}$ : 
Exemple 1. Sensibilisation aux normes du discours rapporté

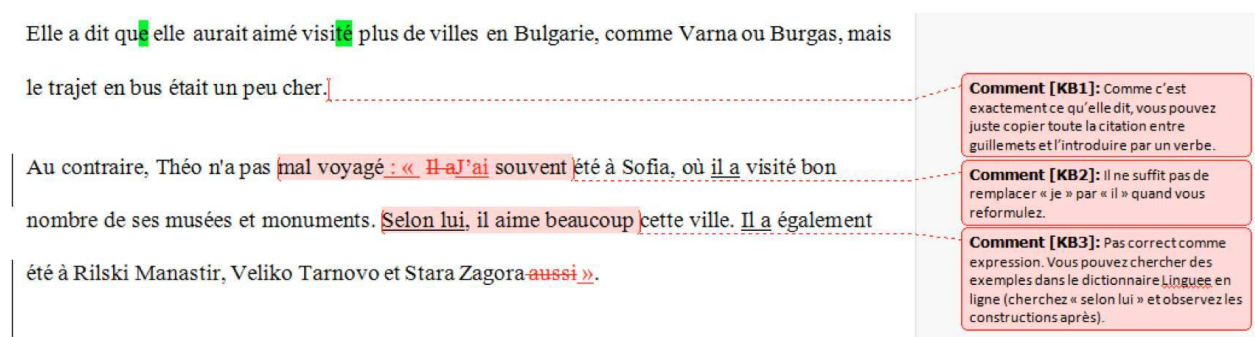

l'enseignant-tuteur permet d'expérimenter et de progresser vers une écriture plus créative et autonome. Des analyses quantitatives confirment cet effet, en relevant des SPA pour certains points langagiers. Par exemple, le taux des rétroactions correctives déclenchées par le feedback varie entre 10 et $26 \%$ des occurrences d'expression de la demande et de connecteurs. Grâce au surlignement des erreurs avec un code de couleurs et aux commentaires renvoyant vers des ressources en ligne (soulignés dans l'exemple suivant), les productions non conformes sont corrigées au moins partiellement ${ }^{4}$ : l'apprenant a proposé «Dans votre site, j'ai lu que vous aideriez les étudiants avec l'hébergement, donc je vous demande si vous pourriez m'aider avec la recherche d'appartement" comme transformation de "스 votre site, j'ai lit que vous aidez les étudiants avec l'hébergement, ainsi je vous demande s'il y a de possibilité de vous m'aider avec la cherche d'appartement ».

Le taux des reprises de l'input déclenchées par la lecture, l'analyse et l'entrainement est entre 33 et $52 \%$. En effet, à l'aide du logiciel de détection du plagiat WCopyfind, il a été possible d'observer le réemploi d'éléments langagiers dans l'article qui résumait trois entretiens avec des locuteurs natifs. L'exemple suivant illustre un extrait de l'article traité avec le logiciel qui insère des hyperliens aux éléments coprésents (voir les mots soulignés) et permet l'affichage parallèle des deux textes ${ }^{5}$ : 
Cécile et Marie sont étudiantes francophones qui sont arrivées en Bulgarie pour étudier $\underline{\text { à }}$ l'AUBG pour la dernière année. Cécile est française, elle fait ses études à Sciences Po Bordeaux. Marie est belge, elle fait un Master en «International Business Management » à Heriot Watt

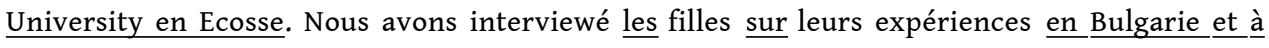
l'AUBG. jusqu'à 5 formules correctes de demande polie et jusqu'à 5 connecteurs adaptés au contexte.

\subsection{Satisfaction des apprenants} tous les considèrent utiles et adaptées à leur niveau et $80 \%$, motivantes. Ils ont apprécié le plus le fait que leur travail était destiné à ou partagé avec des locuteurs natifs. Le deuxième avantage cité est l'exploitation de banques de textes qui les ont aidés à organiser leurs PE et à utiliser des expressions correctes. D'ailleurs, ils trouvent très utile le système d'annotation proposé par l'enseignante-tutrice car il leur permet de se focaliser sur les erreurs. Parmi les fonctionnalités des outils d'aide à la rédaction et à la révision, ils ont classé comme les plus performantes celles des traducteurs contextuels et des correcteurs. Les apprenants utilisent souvent les traducteurs automatiques mais ils sont conscients de certaines imperfections au-delà du mot. Tous sont prêts à recommencer. 


\section{Développement mesuré en Tunisie}

Les résultats, analysés avec SPSS version 21, portent sur l'étude de multiples versions de textes produits et repris par les étudiants. Le choix des éléments observés a été dicté par l'analyse du discours disciplinaire et étoffé par les besoins exprimés par les étudiants. Malgré le nombre limité d'apprenants, les résultats permettent d'une part de dégager un développement généré par des séquences d'interaction favorisant l'acquisition et de mesurer une grande implication. D'autre part, ils mettent en évidence l'impact du dispositif techno-pédagogique d'accompagnement sur les dimensions interdiscursive, discursivo-pragmatiques et transversales de l'écriture. Cet impact se traduit comme suit :

- Au niveau des indicateurs du processus de développement, rétroaction corrective et négociation, des demandes ciblées et personnalisées ont été fréquemment formulées (seul un étudiant n'a pas posé de questions). Sept des 9 étudiants ont maintenu un rythme de négociation assez soutenu pour obtenir une clarification du geste correctif de l'enseignante et/ou une confirmation de la pertinence de leurs reprises. La variété des réponses de la tutrice (avec déclencheur et/ou indicateur ou renvoi à l'input et parfois implicites) a généré des reprises assez diversifiées: seul un étudiant peut reprendre son texte de façon immédiate et naturelle, trois autres le font de manière instable ou différée, une seule ne corrige jamais son écrit et les quatre autres accomplissent les différents types du geste correctif.

- La tâche écrire/réécrire, soutenue/consolidée par l'utilisation de l'input (3 le font souvent, 2 rarement et 3 très rarement) acquiert un potentiel acquisitionnel important. Néanmoins, la paraphrase sous ses diverses formes (copier-coller intégral, copie-coller et reformulation sans citation des sources) témoigne de la difficulté de sortir du copier-coller. Toutefois, pour 5 étudiants cela reste positif pour le développement langagier. Sept étudiants aboutissent à des reformulations certes maladroites mais complètes, incluant citation et normes bibliographiques. Seuls deux étudiants ne respectent pas le processus de réécriture : ajout suppression - remplacement - déplacement. L'effet du tutorat réactif se manifeste : chez la majorité apparaissent une métaréflexion, un recul et une implication dans la tâche, même si l'appropriation ne suit pas toujours immédiatement.

- Les étudiants sont plus sensibles à l'organisation des textes, à la correction de la langue, et tentent de respecter au mieux les normes du genre et de la discipline, mais ils affirment que la surcharge cognitive, la primauté du côté scientifique/disciplinaire et la contrainte temporelle leur font souvent perdre de vue la forme et le sens de leur énoncé. Les résultats le confirment: les erreurs linguistiques (accords/temps/constructions verbales/lexique/ orthographe...) sont corrigées puis parfois refaites par les étudiants. De même, l'emploi des marqueurs du discours et le recours au «nous » de modestie demeurent problématique pour tous.

- L'aspect méthodologique (établir des transitions, introduire, conclure, résumer, traduire...) et les normes de présentation marquent une évolution pour 5 ou 6 étudiants sur 9. Les efforts d'appropriation ont permis de produire des écrits plus complexes sans pour autant atteindre le niveau d'originalité, de variété et de créativité souhaité.

- En ce qui concerne les outils TIC, les résultats révèlent une évolution, néanmoins leur intégration s'avère partielle et leur emploi mérite une réflexion plus approfondie. L'usage des correcteurs se fait sans aucun recul pour la moitié du groupe. La majorité utilise des traducteurs en ligne et se contente du texte proposé (en anglais ou en arabe) sans intervenir pour vérifier si tout est conforme aux attentes du type d'écrit. Ainsi, la complexité et la 
variété des systèmes linguistiques des trois langues (arabe, anglais et français) ne sont pas totalement prises en compte. L'utilisation des logiciels détecteurs de plagiat révèle qu'une pratique réflexive se construit. Chez 4 étudiants, un changement s'opère au niveau des représentations et conduit à de nouveaux automatismes dans le choix des outils et l'appréciation de leur fiabilité. Une apprenante interrompt la réécriture pour chercher l'outil qui lui permette d'augmenter sa créativité au mieux. et évaluées de façon sommative lors du premier semestre (septembre-décembre 2014), ont consisté en l'élaboration de rapports de stage et de miniprojets. La collaboration a permis d'identifier les difficultés et de déterminer les critères d'une grille d'autoévaluation, élaborée par la tutrice et les étudiants qui l'ont consultée et annotée tout le long de la formation. Sa version définitive pourrait être considérée comme une mesure postformation.

\section{Développement mesuré}

Dans ce qui suit, nous aborderons les résultats avec plus de détails et analyserons le développement langagier et les effets du dispositif.

Les données montrent que l'accompagnement (tuteur/apprenant) a permis le passage du guidage à l'autonomie, comme l'indiquent les deuxième et troisième colonnes du tableau 2, ci-après. Les étudiants affirment utiliser les outils TIC pour l'écriture et la réflexion métacognitive avec plus de facilité et trouvent les moyens d'apprendre en s'interrogeant sur leurs pratiques d'écriture même si la quatrième colonne révèle qu'il reste des difficultés. Les données de la dernière colonne nous interpellent car les étudiants se surestiment: les données objectives montrent qu'ils se lancent facilement dans l'action, soutenus par leur estime d'eux-mêmes sans se rendre compte que cela est parfois hâtif. Dans l'ensemble, les étudiants s'impliquent dans ce processus d'évaluation qui leur apprend à distinguer leurs forces et leurs faiblesses, et à agir en conséquence.

Tableau 2. Perception des atteintes des objectifs dans le groupe tunisien

\begin{tabular}{|c|c|c|c|c|}
\hline $\begin{array}{l}\text { Performances } \\
\text { En fin de formation, les étudiants sont } \\
\text { capables de: }\end{array}$ & $\begin{array}{l}\text { Sans } \\
\text { difficultés }\end{array}$ & $\begin{array}{l}\text { Avec } \\
\text { quelques } \\
\text { difficultés }\end{array}$ & $\begin{array}{l}\text { Avec } \\
\text { beaucoup de } \\
\text { difficultés }\end{array}$ & Incapacité \\
\hline $\begin{array}{l}\text { Repérer les passages plagiés en } \\
\text { recourant aux logiciels antiplagiat. }\end{array}$ & 2 & 4 & 3 & Aucun \\
\hline $\begin{array}{l}\text { Choisir des } \\
\begin{array}{l}\text { sources } \\
\text { sélectionner fiables et } \\
\text { pertinentes. }\end{array}\end{array}$ & 5 & 3 & 1 & Aucun \\
\hline $\begin{array}{l}\text { Utiliser un générateur bibliographique } \\
\text { et citer les sources, en respectant les } \\
\text { normes bibliographiques adaptées à la } \\
\text { spécialité. }\end{array}$ & 6 & 2 & 1 & Aucun \\
\hline
\end{tabular}




\begin{tabular}{|c|c|c|c|c|}
\hline $\begin{array}{l}\text { Réécrire en reformulant, en } \\
\text { paraphrasant et en enchainant. }\end{array}$ & Aucun & 5 & 4 & Aucun \\
\hline $\begin{array}{l}\text { S'exprimer objectivement et respecter } \\
\text { les marques de l'énonciation. }\end{array}$ & 2 & 5 & 2 & Aucun \\
\hline $\begin{array}{l}\text { Réviser le texte produit avec un ou des } \\
\text { logiciels de correction. }\end{array}$ & 2 & 3 & 4 & Aucun \\
\hline $\begin{array}{l}\text { Ponctuer et produire des phrases } \\
\text { correctes et claires. }\end{array}$ & 2 & 5 & 2 & Aucun \\
\hline Respecter les délais. & 1 & 6 & 1 & 1 \\
\hline Respecter les normes de mise en page. & 7 & 2 & Aucun & Aucun \\
\hline
\end{tabular}

L'étude du corpus montre que ne pas fournir à l'apprenant un modèle correct dans la langue cible peut donner des résultats positifs sur le plan métacognitif car l'étudiant se prend en charge et se corrige ensuite. De surcroit, les commentaires portant sur le succès des reformulations encouragent et conduisent l'étudiant à s'impliquer d'avantage en contrôlant ses connaissances et ses pratiques. Il prend souvent conscience de l'inadéquation entre son texte et les normes professionnelles et académiques et réfléchit avec la tutrice à une nouvelle démarche en anticipant les conséquences de ses choix, comme l'illustre l'exemple suivant, extrait d'un commentaire, rédigé par un étudiant sur Google drive: "j'ai définit le concept selon mes propres mots, pourquoi les []?... c'est des connaissances basics madame... mais je dois mettre les sources?».

L'input auquel les apprenants sont exposés est repris avec l'aide de la tutrice mais, parfois, la médiation est à l'initiative des pairs. Les forums facilitent une évaluation formative mutuelle : «j'ai vu que le correcteur parfois, il n'accepte pas quelque mot maisj'ai pas trouvé la réponse pourquoi », «Mais R. cela ne t'étonne pas que Viper n'a pas relevé que tu utilises 4 fois la même référence avec le même $n^{\circ}$ ?", " Mais, j'ai essayé de scanner le même document sans la référence et VIPER m'affiche la source de la copie coller, ainsi qu'il compte les mots et affiche le pourcentage du plagiat. "

50 L'activité de relecture-commentaire des textes réécrits, accomplie en présentiel, face à la tutrice et aux pairs, pointe les difficultés à surmonter comme l'illustre cet extrait d'une séquence de verbalisation: "Le passage contenait des mots qui portent des liens hypertextes laissés après ... [émoticône sourire] opération de "copie/coller"... j'ai aussi oublié de... la suppression de la mise en forme source... Maintenant c'est corrigé ».

51 Toutefois, le développement est irrégulier car, malgré le travail accompli, produire des phrases complètes et claires reste ardu. Certains se contentent de déplacer une phrase où il n'y a pas de verbe conjugué, au lieu de la corriger. Pour d'autres, il s'avère vraiment difficile de réécrire en reformulant et en respectant les normes bibliographiques adaptées à la spécialité. Ils se limitent à l'ajout de la référence, sans reformuler ou mettre les guillemets ou encore prendre du recul par rapport au texte de départ, comme le révèle l'exemple suivant: "Cette nouvelle technologie parait être la révélation qui va vous permettre d'être les plus performants et de gérer le coût de vos systèmes d'information plus sereinement [15]. » Malgré l'intervention de la tutrice, qui avait surligné (ici souligné) les indices de plagiat 
et avait invité l'apprenant à apporter les modifications voulues, celui-ci n'a fait qu'indiquer la source.

De surcroit, la réflexion métacognitive est entravée par le manque de confiance en soi qui amène le seul binôme du groupe à se rassurer avec le logiciel antiplagiat qui ne prend pas nécessairement en compte toutes les phrases copiées et collées intégralement. De plus, ces étudiantes font un choix paradoxal en recourant à wikipédia: «je ne trouve pas des informations valides, pour cela je l'utilise wikipédia comme une source ».

\section{Satisfaction des étudiants}

53 Le dépouillement des questionnaires de satisfaction recueillis à la fin de la formation a permis une analyse interprétative des réponses qui a révélé que la majorité des étudiants confirment la progression des pratiques et l'impact positif du dispositif technopédagogique sur l'écriture/réécriture et sur la réflexion métacognitive :

54 «J'ai opté vers CHICAGO qui m'offre les différentes normes selon le type du support (livre, ouvrage, extrait de livre, journal, article...)»

"Avec l'exercice et l'aide de Madame et des correcteurs orthographiques et de grammaire j'y arrive 》

56 "Oui, j'ai utilisé et continue à utiliser CORDIAL, word parfois reverso pour réviser ce que j'écris. ». Ici, l'étudiante affirme avoir eu recours à plusieurs correcteurs linguistiques afin de rédiger un écrit d'un niveau élevé de correction et de complexité. Cette variété dévoile une quête de perfection. Son intention de maintenir cette pratique souligne sa satisfaction.

Cependant, pour quelques-uns, les normes individuelles et celles qu'ils attribuent aux enseignants divergent, comme le montrent les exemples suivants: «... trop difficile la reformulation... et il faut vérifier a chaque fois la source... c'est long... je perd trop de temp... ». Les mots clés ici sont «difficile» et "long»: le temps consacré à la tâche représente une contrainte supplémentaire pour cet étudiant.

Quand un apprenant écrit : «Le copier/coller, tout le monde le fait... c'est plus simple... et on fait moins de faute. » il montre qu'il maintient ses anciennes pratiques. Ce qui se comprend au $\mathrm{vu} d u$ poids des représentations et de l'aspect sécurisant du copier-coller sans reformulation qui reste propice au développement si l'on s'appuie sur A. EiseleHenderson (2000). Même si les critères sociaux et les critères cognitifs sont ici en contradiction, se confirme ici la convergence du développement ressenti/effectif qu'il est possible de considérer comme un indicateur de l'impact positif de l'action menée.

\section{Discussion générale}

59 Au-delà de leurs limites, ces deux expériences confirment les résultats de l'expérience sur l'emploi du copier-coller (Narcy-Combes, 2005). Le lexique se stabilise progressivement et certains éléments de morphosyntaxe se développent en fonction des tâches et des thèmes proposés et non d'une progression pré-établie. Les résultats bulgares soutiennent particulièrement l'hypothèse $d u$ développement par blocs langagiers (instances/ collocations). 

stratégies qu'ils élaborent, les évaluent et en adoptent progressivement d'autres. Par contre, les risques de plagiat impliquent une réflexion approfondie. La langue de scolarisation cède progressivement la place à la langue cible comme code fournisseur, surtout si de l'input est fourni sous forme de banques de textes correspondant aux tâches, thèmes et exigences spécifiques des domaines scientifiques ou techniques. Il y a passage progressif entre la traduction, la réadaptation et la recréation. Ce passage déclenche des processus de développement et permet de réduire, puis de court-circuiter, le recours aux outils disponibles dont l'emploi se rationalise. C'est la réflexion méta, individuelle et collective qui semble connaitre le développement le plus remarquable. Elle conduit les apprenants à poser des questions au tuteur ou à leur(s) pair(s) pendant la phase d'écriture et à débattre des solutions parfois sur des points généraux mais également sur des problèmes spécifiques. Par contre, elle peut devenir une surcharge cognitive, ce qui confirme les résultats d'autres recherches (cf. Roehr, 2014) et impose de la moduler.

Notre option d'appliquer à ce type d'écriture une recherche de séquences que nous pouvons qualifier de SPA se trouve confirmée par le fait qu'elles sont relativement nombreuses, comme le sont les reprises de l'input dans la production (output compréhensible), parfois sous forme purement copiée (voir les données tunisiennes). Lorsque le tuteur propose des apports correctifs, les effets ne sont pas nécessairement bénéfiques (données tunisiennes). Cela s'explique par les difficultés relatives au genre du discours à produire et pourrait indiquer qu'il importe que les problèmes soient ressentis par les apprenants eux-mêmes, mais les résultats bulgares sont plus positifs sur ce point. La créativité se développe, et les aspects pragmatico-discursifs s'ajustent aux attentes. Quand des textes ressources sont proposés les apprenants les apprécient pour structurer leurs écrits de manière adéquate et y puiser mots et constructions utiles, même si la reformulation pose encore quelques problèmes.

L'expérience bulgare a mis en relief le rôle du travail collaboratif qui met les apprenants dans une optique de communication et dans le regard de l'autre. Le wiki fonctionne comme un véritable outil en ligne d'organisation du groupe. Il sert de cahier de textes et de carnet de bord. Sa fonctionnalité « historique dynamique » permet l'accès aux diverses versions et donne la possibilité d'observer les processus rédactionnels. Dans la mesure où la recherche montre que l'appartenance à une communauté discursive régulerait efficacement le lexique, l'orthographe et la grammaire ${ }^{6}$, le recours à des tâches sociales trouve ainsi une justification alors que des tâches purement scolaires ne déclenchent pas la même attention aux normes. En Bulgarie, le fait de publier les articles-témoignages sur un site et de communiquer par courriel avec des locuteurs natifs a rendu les étudiants plus attentifs à la correction de leurs textes qu'ils ne l'étaient pour accomplir des tâches de simulation et, en Tunisie, l'objectif des apprenants est de valider leurs études et de construire une image professionnelle, ce qui justifie le travail.

Certes, le tuteur peut reprendre plus de détails que les pairs, mais lorsque les apprenants écrivent beaucoup, il court le risque d'être submergé si ces derniers ne sont pas entrainés au travail de révision que permet le numérique.

Les apprenants évaluent positivement les dispositifs et les tâches qui leur paraissent utiles, adaptées à leur niveau, et motivantes. Ils se sentent peu bridés et pensent que leur PE a progressé en termes de correction, d'adéquation et de complexité grâce au travail d'entrainement et qu'ils sont devenus plus attentifs à l'organisation des textes. Ils apprécient la possibilité de remettre plusieurs versions des PE, ou de pouvoir les 
reprendre, car cela leur permet de se focaliser sur les problèmes et de les résoudre seuls (SPA). Tous accepteraient de participer de nouveau à des tâches de ce genre.

$\mathrm{Au}$ vu de la fréquence des SPA, des reprises de l'input dans les écrits, de la réflexion métacognitive, de l'implication des apprenants dans les expériences, il est possible de conclure qu'un travail qui s'appuie sur le déjà-là langagier et méthodologique des apprenants favorise le développement de la production écrite et conduit à produire des textes qui (1) répondent de façon satisfaisante aux attentes institutionnelles, ou (2) compensent en partie les objectifs peu spécifiques de certaines formations. Les résultats confirment que les apprenants sont en train de développer un système de contrôle souple qui permet de manipuler leurs propres ressources langagières et les aides à la rédaction et à la révision externes de manière à atteindre une complexité et correction plus grandes de leur expression écrite.

\section{Limites et biais}

Il s'agit d'interventions, d'une durée limitée, et non de recherche quasi-expérimentale en acquisition, et l'attention se porte autant sur le dispositif que sur les données (Dörnyei, 2007). Les résultats mériteraient d'être confirmés par d'autres études ou des recherches plus directement acquisitionnelles. Il est difficile de faire des hypothèses au sujet de leur durabilité si, en ce qui concerne les aspects discursivo-pragmatiques, un changement durable n'est observable qu'au terme d'une pratique assez conséquente (DuFon, 2010).

Les outils en ligne ont été utiles pour trouver des constructions ayant une fonction langagière spécifique, mais peu efficaces pour faire repérer les productions non conformes du point de vue des normes sociolinguistiques, ce qui peut créer quelques problèmes alors que les interactions qui se créent avec des locuteurs natifs, les pairs et l'enseignant-tuteur permettent mieux de réfléchir sur ces normes (cf. DuFon, 2010).

Les apprenants ne sont pas toujours préparés pour le travail collaboratif. Leur résistance se manifeste surtout lorsqu'ils doivent écrire des textes longs ou corriger les productions des autres. Dans le premier cas, le problème vient de la contribution inégale des membres des groupes. Dans le second, elle est due au manque de confiance dans leurs propres connaissances.

\section{Conclusion}

Pour le moment, les dispositifs dépendent de l'enseignant qui les met en place, et la lourdeur de la charge ne saurait être dissimulée. L'expérience bulgare met en relief le rôle du travail collaboratif et le rôle organisateur des dispositifs numériques qui facilite la tâche du tuteur, ce qui correspond aux résultats d'autres expériences en communication médiée par ordinateur (cf. Lamy \& Hampel, 2007). L'appartenance à une communauté discursive semble avoir une influence positive sur le désir d'y participer et d'en respecter les normes: créer des groupes de travail internationaux aurait certainement des effets positifs.

70 Il reste à développer une compréhension affinée du potentiel des outils disponibles et de l'importance du contenu qui peut expliquer la réussite en Tunisie (spécificité du contenu). 
71 Le rôle du tuteur nécessite une réflexion approfondie. Dans le cas de tâches complexes, le rôle de l'accompagnement pour aider les apprenants à gérer le temps et les activités aux différentes étapes est très important. Les tâches collaboratives d'entrainement préparant les tâches sociales pourraient jouer un rôle aussi efficace que le feedback indirect de l'enseignant-tuteur. Pour créer des SPA et favoriser le repérage des écarts, il convient donc de savoir comment « doser » les divers types de tâches.

Nous n'avons pas abordé les problèmes d'équipement, mais nous y sommes particulièrement sensibles. Par contre, le pari que nous avons fait de nous appuyer sur le déjà-là langagier et méthodologique des apprenants nous a permis de créer des circonstances d'apprentissage qui motivent et autonomisent les apprenants, ce qui est un premier pas encourageant.

\section{BIBLIOGRAPHIE}

BERTIN, J-C., GRAVÉ, P. \& NARCY-COMBES, J.-P. (2010). Second Language Distance Learning. Theoretical Perspectives and Didactic Ergonomics. Hershley, PA : IGI Global.

BIASI, P-M. de (2011). Génétique des textes. Paris : CNRS éd.

BUSSIÈRE, J. (2013). Utiliser les traducteurs automatiques en ligne pour développer l'autonomie en production écrite en langue étrangère. Mémoire de Master 2 Recherche : Université Sorbonne nouvelle-Paris 3.

CIEKANSKI, M. (2014). « Accompagner l'apprentissage des langues à l'heure du numérique Évolution des problématiques et diversité des pratiques ». Alsic 17. En ligne : http:// alsic.revues.org/2762, consulté le 04 juillet 2015.

cook, V. (2008). Second Language Learning and Language Teaching. London : Arnold.

DE PIETRO, J.-F., MATTHEY, M. \& PY, B. (1989). « Acquisition et contrat didactique : les séquences potentiellement acquisitionnelles dans la conversation exolingue ». In : Actes du troisième Colloque Régional de Linguistique, 28-29 avril 1988. Strasbourg : Université Louis Pasteur, p. 99-119.

DÖRNYEI, Z. (2007). Research Methods in Applied Linguistics. Oxford : OUP.

DUFON, M.A. (2010). « The acquisition of terms of address in a second language ». In : Trosborg, A. (éd.) (2010), Pragmatics across Languages and Cultures (Handbooks of Pragmatics). Berlin : Walter de Gruyter, p. 309-332.

EISELE-HENDERSON, A. (2000). La lecture de textes en anglais langue étrangère chez des étudiants en sociologie. Implications didactiques d'une perspective socio-cognitive. Thèse de doctorat : Université de Savoie.

ELLIS, N. (1993). « Rules and Instances in Foreign Language Learning: Interactions of Explicit and Implicit Knowledge ». European Journal of Cognitive Psychology 5(3), p. 289-318.

GAONAC'H, D. (1990). Acquisition et utilisation d'une langue étrangère. Paris : Hachette. 
GARCIA, O. (2009). Bilingual education in the 21st century: A global perspective. Chichester, England : Wiley- Blackwell.

GOES, J., MANGIANTE, J-M. \& BORDO, W. (2016). Le Français sur Objectif Universitaire. Entre apports théoriques et pratiques de terrain. Arras : Artois presses université.

HERDINA, P. \& JESSNER, U. (2002). A dynamic Model of Multilingualism, Perspectives of Change in Psycholinguistics. Clevedon : Multilingual Matters.

LAMY, M.-N. \& HAMPEL, R. (2007). Online Communication in Language Learning and Teaching. Basingstoke : Palgrave McMillan.

LOGAN, G. (1988). « Towards an instance theory of automatisation ». Psychological Review 95, p. $492-527$.

LOISY, C., CHARNET, C. \& RIVENS MOMPEAN, A. (2011). « Pratiques d'écriture en ligne pour l'apprentissage des langues ». Revue internationale des technologies en pédagogie universitaire 8(1-2), p. 58-68.

MANGIANTE, J-M. \& PARPETTE, C. (2011). Le français sur objectif universitaire. Grenoble : Presses universitaires de Grenoble.

NARCY-COMBES, J.-P. (2005). Didactique des langues et TIC. Paris : Ophrys.

O'BRIEN, T. (2004). « Writing in a foreign language: teaching and learning ». Language Teaching 37 (1), p. 1-28.

PICA, T., KANG, H.-S. \& SAURO, S. (2006). « Information gap tasks: Their multiple roles and contributions to interaction research methodology ». Studies in Second Language Acquisition 28(2), p. 301-338.

RANDALL, M., (2007). Memory, psychology and second language learning. Amsterdam-Philadelphia : John Benjamins.

ROEHR, K. (2014). « Metalinguistic Knowledge and Language Ability in University-level L2 Learners ». Applied Linguistics 29(2), p. 173-199.

STORCH, N. (2013). Collaborative Writing in L2 Classrooms. Bristol : Multilingual Matters.

SWAIN, M. \& LAPKIN, S. (1995). « Problems in output and the cognitive processes they generate: A step towards second language learning ». Applied Linguistics 16, p. 371-391.

WILLIAMS, C. (2002). Extending Bilingualism in the Education System. Education and Lifelong Learning Committee Report. En ligne : http:// www.assemblywales.org/3C91C7AF00023D820000595000000000.pdf.

WILLIAMS, S. \& HAMMARBERG, B. (1998). « Language Switches in L3 Production: Implications for a Polyglot Speaking Model ». Applied Linguistics 19(3), p. 295-333.

WONG, W. \& SIMARD, D. (2001). « La saisie, cette grande oubliée ! ». Acquisition et interaction en langue étrangère 14. En ligne : http://aile.revues.org/1476.

WRAY, A. (2008). Formulaic language: pushing the boundaries. Oxford : Oxford University Press. 


\section{NOTES}

1. Par input on entend l'information produite dans la langue cible à laquelle l'apprenant est exposé, l'intake est la partie de l'input intégrée ou intériorisée par l'apprenant et l'output ce qu'il produit (voir Wong et Simard, 2001 par ex.).

2. «... a natural way of developing and strengthening both languages whilst simultaneously gaining a deeper understanding " (notre traduction).

3. Dans les exemples, les prénoms sont anonymisés en fonction de la nationalité des participants.

4. « Aider avec » reste une activation partielle de l'anglais.

5. Le logiciel n'affiche pas les correspondances à $100 \%$, donc une relecture attentive s'impose.

6. L'efficacité de l'interaction entre pairs pour améliorer la qualité des productions écrites est mise en évidence par des recherches (O'Brien, 2004). Chacun apprend en commentant le travail des autres, le feedback des pairs est disponible immédiatement et correspond plus aux aptitudes des apprenants que la rétroaction de l'enseignant (Storch, 2013). Les situations de résolution de problème, de négociation de sens et de forme assurent mieux le repérage que les interactions traditionnelles en classe de langue (Pica, Kang \& Sauro, 2006). Dans l'interaction en ligne avec d'autres apprenants ou des locuteurs natifs, l'intervention de l'enseignant peut avoir un effet négatif sur les échanges (Loisy, Charnet \& Rivens Mompean, 2011).

\section{RÉSUMÉS}

Considérer la production écrite en tant qu'activité individuelle monolingue empêche souvent les apprenants de langue 2 ou 3 de laisser libre cours à leur créativité et d'atteindre la complexité et la correction souhaitées. Les fondements théoriques qui expliquent l'écriture comme un processus récursif, multimodal et bi-/plurilingue, ainsi que quelques expériences initiales menées sur le terrain ont révélé que des dispositifs et tâches qui favorisaient ces aspects déclenchaient un développement langagier positif. La présente contribution synthétise deux expériences de recherche-action visant à étayer la production écrite en français dans des contextes universitaires bulgare et tunisien en s'appuyant sur le potentiel des technologies de l'information et de la communication. Malgré les différences des contextes et des caractéristiques du public, les résultats convergent : le dispositif qui organise dans un ensemble cohérent l'accompagnement par le tuteur, les outils et les ressources numériques, la collaboration et l'interaction avec des pairs ou des locuteurs natifs, favorise l'amélioration progressive de la production écrite, la sensibilisation aux normes, la créativité et l'autonomisation des apprenants.

If written production is seen as an individual monolingual activity, L2 or L3 learners are often unable to be creative and to develop accuracy and complexity as expected. Research describing writing as a recursive, multimodal and bi/plurilingual process and some initial practical experiments have shown that learning environments and tasks favouring these aspects were conducive to language development. This contribution is a synthetic description of two research action experiments in collaborative blended courses of written French in higher education contexts in Bulgaria and Tunisia with a wide and planned use of the resources provided by 
Information and Communication Technology (ICT). Though the contexts and the students were different, results are very similar: written production improves in a learning environment where tutor mediation, digital tools and resources, collaboration and interaction among peers and native speakers of the target language are organized in a coherent system. Sensitization to norms, creativity and learner autonomy are also shown to improve.

INDEX

Mots-clés : production écrite, langue 2, alternance codique, tutorat, technologies de l'information et de la communication

Keywords : written production, L2, code switching, tutoring, ICT

\section{AUTEURS}

\section{KRASTANKA BOZHINOVA}

American University in Bulgaria, Université de Nantes, Crini, EA 1162, F-44000, France

\section{JEAN-PAUL NARCY-COMBES}

Université Sorbonne Nouvelle - Paris 3, Diltec, EA 2288, F-75005, France

\section{SONIA ZAOUALI}

Institut Supérieur de l'Éducation et de la Formation Continue de Tunis, Université Sorbonne Nouvelle - Paris 3, Diltec, EA 2288, F-75005, France 